ERR A T UM

Sofia De Gregorio · Silvio G. Rotolo · Igor M. Villa

\title{
Geochronology of the medium to high-grade metamorphic units of the Peloritani Mts., Sicily
}

Published online: 29 January 2004

(C) Springer-Verlag 2004

\section{Int J Earth Sci (Geol Rundsch) 98:852-872}

Due to an unfortunate error the last column of Table 5 is missing. The correct table is printed below.

\section{Appendix}

(see next page)

The online version of the original article can be found at http:// dx.doi.org/10.1007/s00531-003-0356-5

S. De Gregorio $(\varangle) \cdot$ S. G. Rotolo

Dipartimento di Chimica e Fisica della Terra (CFTA),

Università di Palermo,

Via Archirafi, 36-90123 Palermo, Italy

e-mail: sofiadg@tiscalinet.it

Fax: +39-091-6168376

I. M. Villa

Isotopengeologie,

Erlachstrasse 9a, 3012 Bern, Switzerland

I. M. Villa

Dipartimento di Scienze Geologiche e Geotecnologie,

Università di Milano,

Bicocca, Italy 
Table 5 Ar-Ar stepwise heating results. All Ar concentrations are given in picolitres per gram ( $\mathrm{pL} / \mathrm{g})$; errors are $1 \sigma$; $\mathrm{Ar}^{*}$ denotes total ${ }^{40} \mathrm{Ar}$ minus atmospheric ${ }^{40} \mathrm{Ar}$. The integrated values of $\mathrm{K}(\mathrm{wt} \%$ o) $\mathrm{Ca}$ (wt $\%$ ) and $\mathrm{Cl}(\mathrm{ppm})$ were calculated from the total ${ }^{39} \mathrm{Ar},{ }^{37} \mathrm{Ar}$ and ${ }^{38} \mathrm{Ar}$, the production ratios and the irradiation time. Ages are given in $\mathrm{Ma}$

\begin{tabular}{|c|c|c|c|c|c|c|c|}
\hline Step & $\begin{array}{l}\mathrm{T} \\
\left({ }^{\circ} \mathrm{C}\right)\end{array}$ & ${ }^{40} \mathrm{Ar}$ tot & ${ }^{39} \mathrm{Ar}$ & ${ }^{38} \mathrm{Ar}$ & ${ }^{37} \mathrm{Ar}$ & ${ }^{36} \mathrm{Ar}$ & Age $\pm 1 \sigma$ \\
\hline
\end{tabular}

Mela Unit

CRO 1 hbl (33.9 mg); J=2.842* 10-3; Ar*=3,090; K=2.1\%o; Ca=7.6\%; Cl=108 ppm

$\begin{array}{rrrll}1 & 717 & 248.10 & \pm & 0.04 \\ 2 & 923 & 123.99 & \pm & 0.02 \\ 3 & 981 & 415.31 & \pm & 0.03 \\ 4 & 1,011 & 1,011.00 & \pm & 0.11 \\ 5 & 1,041 & 559.96 & \pm & 0.04 \\ 6 & 1,065 & 99.50 & \pm & 0.04 \\ 7 & 1,108 & 180.16 & \pm & 0.04 \\ 8 & 1,146 & 85.45 & \pm & 0.04 \\ 9 & 1,280 & 482.20 & \pm & 0.06 \\ 10 & 1,383 & 227.25 & \pm & 0.04\end{array}$

\begin{tabular}{|c|c|c|c|}
\hline 0.537 & 0.007 & 0.888 & 0.005 \\
\hline 1.311 & 0.005 & 1.593 & \pm 0.011 \\
\hline 5.121 & 0.008 & 1.506 & \pm 0.007 \\
\hline 13.343 & 0.012 & 2.266 & \pm 0.007 \\
\hline 8.127 & 0.010 & 0.728 & \pm 0.007 \\
\hline 1.216 & 0.005 & 0.284 & \pm 0.006 \\
\hline 2.301 & 0.007 & 0.479 & \pm 0.006 \\
\hline 0.986 & 0.006 & 0.248 & \pm 0.007 \\
\hline 6.103 & 0.009 & 1.393 & \pm 0.007 \\
\hline 3.065 & 0.005 & 0.556 & 0.006 \\
\hline
\end{tabular}

$\begin{aligned} 5.22 & \pm \\ 21.32 & \pm \\ 101.97 & \pm \\ 268.35 & \pm \\ 158.99 & \pm \\ 24.03 & \pm \\ 47.40 & \pm \\ 20.13 & \pm \\ 121.26 & \pm \\ 53.51 & \pm\end{aligned}$

$0.07 \quad 0.532 \pm 0.006$

$0.10 \quad 0.092 \pm 0.005$

$0.30 \quad 0.138 \pm 0.006$

$0.720 .165 \pm 0.006$

$0.48 \quad 0.099 \pm 0.004$

$0.130 .046 \pm 0.005$

$0.190 .046 \pm 0.005$

$0.140 .051 \pm 0.004$

$\begin{array}{lll}0.36 & 0.104 & \pm \\ 0.002\end{array}$

$0.17 \quad 0.098 \pm 0.005$

$715.8 \pm 14.0$

$352.6 \pm 4.5$

$351.3 \pm 1.4$

$347.1 \pm 0.5$

$317.4 \pm 0.5$

$340.6 \pm 4.4$

$348.7 \pm 2.3$

$344.4 \pm 5.0$

$355.0 \pm 0.6$

CRO 2 bt $(2.2 \mathrm{mg}) ; \mathrm{J}=2.845^{*} 10-3 ; \mathrm{Ar} *=53,000 ; \mathrm{K}=74.1 \%$; $\mathrm{Ca}=0.2 \% ; \mathrm{Cl}=1.474 \mathrm{ppm}$

\begin{tabular}{|c|c|c|c|c|c|c|c|c|c|c|c|c|c|c|}
\hline 539 & $5,156.30$ & \pm 1.00 & 89.151 & \pm 0.095 & 11.832 & \pm 0.080 & 1.11 & \pm & 0.26 & 5.87 & \pm & 0.11 & 187.0 & \pm \\
\hline 631 & $6,208.60$ & \pm 1.80 & 119.14 & \pm 0.12 & 12.258 & \pm 0.105 & 1.01 & \pm & 0.18 & 2.03 & \pm & 0.12 & 226.9 & \pm \\
\hline 710 & $10,236.40$ & \pm 1.55 & 206.33 & \pm 0.21 & 17.750 & \pm 0.110 & 0.86 & \pm & 0.23 & 0.87 & 1 & 0.11 & 232.7 & \pm \\
\hline 760 & $6,991.70$ & \pm 0.80 & 141.11 & \pm 0.14 & 14.412 & \pm 0.090 & 0.87 & \pm & 0.15 & 0.948 & \pm & 80 & 229.0 & \pm \\
\hline 832 & $4,870.25$ & \pm 0.90 & 97.90 & \pm 0.13 & 11.362 & \pm 0.135 & 0.00 & \pm & 0.00 & 0.21 & - & 0. & 6.0 & \pm \\
\hline 875 & $6,565.15$ & \pm 1.00 & 132.58 & \pm 0.18 & 14.486 & \pm 0.075 & 0.37 & \pm & 0.30 & 0.767 & 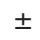 & 0 . & 230.1 & \pm \\
\hline 917 & $8,636.35$ & \pm 1.80 & 174.98 & \pm 0.19 & 18.618 & \pm 0.095 & 1.40 & \pm & 0.17 & 1.001 & 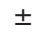 & 0.055 & 229.5 & \pm \\
\hline 956 & $6,984.45$ & \pm 1.55 & 138.59 & \pm 0.16 & 12.563 & \pm 0.085 & 2.14 & \pm & 0.18 & 1.574 & - & 0.090 & 226.7 & \pm \\
\hline 015 & $4,012.09$ & \pm 1.25 & 74.87 & \pm 0.080 & 5.428 & \pm 0.100 & 0.00 & \pm & 0.00 & 0.97 & $I$ & 0.10 & 239.0 & \pm \\
\hline 50 & $2,563.47$ & \pm 1.15 & 41.71 & \pm 0.12 & 3.498 & \pm 0.085 & 2.95 & \pm & 0.30 & 1.811 & $I$ & 0.090 & 233.9 & \pm \\
\hline 1396 & $1,791.60$ & \pm 1.60 & 18.18 & \pm 0.10 & 2.540 & \pm 0.105 & 8.04 & \pm & 0.23 & 3.301 & $\perp$ & 0.095 & 217.1 & \pm \\
\hline
\end{tabular}

FR $1 \mathrm{hbl}(16.5 \mathrm{mg}) ; \mathrm{J}=6.600 * 10-3 ; \mathrm{Ar} *=4,270 ; \mathrm{K}=3.6 \%$; $\mathrm{Ca}=7.0 \% ; \mathrm{Cl}=61 \mathrm{ppm}$

\begin{tabular}{|c|c|c|c|c|}
\hline 630 & 157.92 & \pm 0.02 & $1.143 \pm 0.008$ & $0.370 \pm 0.015$ \\
\hline 750 & 77.28 & \pm 0.02 & $0.486 \pm 0.016$ & $0.172 \pm 0.013$ \\
\hline 910 & 269.89 & \pm 0.04 & $7.504 \pm 0.016$ & $3.265 \pm 0.015$ \\
\hline 950 & 461.21 & \pm 0.08 & $14.795 \pm 0.022$ & $1.901 \pm 0.010$ \\
\hline 980 & 1380.79 & \pm 0.13 & $47.998 \pm 0.046$ & $2.520 \pm 0.014$ \\
\hline 1,000 & 490.93 & \pm 0.05 & $16.343 \pm 0.022$ & $0.822 \pm 0.015$ \\
\hline 1,040 & 195.94 & \pm 0.05 & $5.755 \pm 0.010$ & $0.577 \pm 0.017$ \\
\hline 1,080 & 335.99 & \pm 0.05 & $10.658 \pm 0.016$ & $0.875 \pm 0.018$ \\
\hline 170 & $1,201.88$ & \pm 0.13 & $40.268 \pm 0.040$ & $2.936 \pm 0.013$ \\
\hline 1,38 & 657.61 & \pm 0.29 & $19.496 \pm 0.022$ & 1.392 \\
\hline
\end{tabular}

FR 2 hbl (30.4 mg); J=2.833* 10-3; $\mathrm{Ar} *=2,870 ; \mathrm{K}=2.1 \%$; $\mathrm{Ca}=6.9 \% ; \mathrm{Cl}=32 \mathrm{ppm}$

$\begin{array}{rrrrrrrrr}1 & 701 & 173.92 & \pm 0.03 & 1.000 \pm 0.007 & 0.261 & \pm & 0.003 \\ 2 & 911 & 110.41 & \pm 0.03 & 1.440 \pm 0.006 & 0.155 & \pm & 0.006 \\ 3 & 954 & 267.33 & \pm 0.03 & 3.404 \pm 0.007 & 0.268 & \pm & 0.008 \\ 4 & 973 & 378.70 & \pm 0.06 & 5.347 \pm 0.011 & 0.400 & \pm & 0.005 \\ 5 & 990 & 317.98 & \pm & 0.03 & 4.557 \pm 0.008 & 0.294 & \pm & 0.008 \\ 6 & 1,009 & 100.96 & \pm & 0.04 & 1.336 \pm 0.006 & 0.103 & \pm & 0.007 \\ 7 & 1,027 & 63.43 & \pm & 0.04 & 0.769 \pm 0.006 & 0.097 & \pm & 0.005 \\ 8 & 1,063 & 174.06 & \pm & 0.06 & 2.397 \pm 0.005 & 0.174 & \pm & 0.006 \\ 9 & 1,148 & 640.89 & \pm & 6.58 & 8.958 \pm 0.089 & 0.651 & \pm & 0.007 \\ 10 & 1,282 & 729.93 & \pm & 0.07 & 10.202 \pm 0.011 & 0.743 & \pm & 0.006 \\ 11 & 1,380 & 219.79 & \pm & 0.08 & 2.853 \pm 0.007 & 0.198 & \pm & 0.006\end{array}$

FR 3 hbl (12.7 mg); J=6.600* 10-3; $\mathrm{Ar}^{*}=6,590 ; \mathrm{K}=5.5 \%$; $\mathrm{Ca}=7.7 \% ; \mathrm{Cl}=93 \mathrm{ppm}$

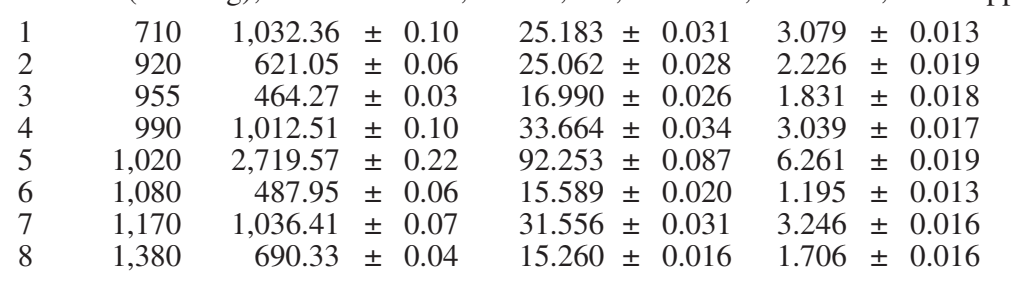

$\begin{aligned} 1.31 & \pm \\ 1.04 & \pm \\ 3.46 & \pm \\ 7.78 & \pm \\ 22.30 & \pm \\ 7.68 & \pm \\ 5.05 & \pm \\ 6.14 & \pm \\ 24.21 & \pm \\ 11.35 & \pm\end{aligned}$

$11.35 \pm$

$4.39 \pm$

$19.90 \pm$

$67.92 \pm$

$101.48 \pm$

$81.97 \pm$

$23.94 \pm$

$14.67 \pm$

$43.81 \pm$

$156.76 \pm$

$178.54 \pm$

$48.82 \pm$ $\begin{array}{llll}0.21 & 0.572 & \pm & 0.010 \\ 0.18 & 0.256 & \pm & 0.007 \\ 0.30 & 0.342 & \pm & 0.006 \\ 0.29 & 0.273 & \pm & 0.011 \\ 0.24 & 0.360 & \pm & 0.013 \\ 0.30 & 0.215 & \pm & 0.007 \\ 0.20 & 0.217 & \pm & 0.013 \\ 0.29 & 0.208 & \pm & 0.012 \\ 0.22 & 0.362 & \pm & 0.008 \\ 0.18 & 0.474 & \pm & 0.011\end{array}$

$0.040 .336 \pm 0.006$

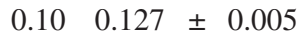

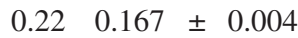

$\begin{array}{llll}0.34 & 0.045 & \pm & 0.009\end{array}$

$0.250 .052 \pm 0.006$

$0.120 .030 \pm 0.006$

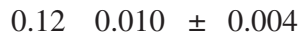

$0.140 .038 \pm 0.007$

$2.260 .150 \pm 0.002$

$0.500 .171 \pm 0.005$

$\begin{array}{lll}0.18 & 0.106 \pm 0.004\end{array}$
$2.75 \pm$ $9.85 \pm$ $14.02 \pm$ $48.60 \pm$ $184.48 \pm$ $41.70 \pm$ $77.16 \pm$ $33.42 \pm$ $\begin{array}{lll}1.13 & 1.715 & \pm \\ 1.06 & 0.018\end{array}$

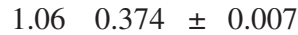

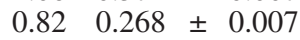
$1.610 .381 \pm 0.009$ $0.920 .596 \pm 0.009$ $1.40 \quad 0.316 \pm 0.013$ $\begin{array}{llll}1.73 & 0.530 & \pm & 0.014\end{array}$ $0.660 .854 \pm 0.006$ $\begin{array}{rlr}-18.0 & \pm & 32.0 \\ 78.2 & \pm & 49.0 \\ 257.8 & \pm & 2.4 \\ 292.4 & \pm & 2.0 \\ 299.6 & \pm & 0.6 \\ 295.7 & \pm & 1.2 \\ 269.6 & \pm & 7.2 \\ 293.6 & \pm & 2.9 \\ 308.6 & \pm & 0.5 \\ 301.8 & \pm & 1.5\end{array}$

$348.4 \pm 7.2$ $248.4 \pm 4.9$

$311.2 \pm 1.6$ $329.3 \pm 1.1$

$320.4 \pm 1.0$

$330.9 \pm 4.5$

$372.8 \pm 4.6$

$327.2 \pm 2.5$

$321.0 \pm 4.2$

$320.8 \pm 0.6$

$318.4 \pm 1.8$

$233.2 \pm 2.2$ $229.1 \pm 0.9$ $254.8 \pm 1.3$ $299.2 \pm 0.8$ $310.1 \pm 0.3$ $290.0 \pm 2.1$ $315.1 \pm 1.2$ $322.2 \pm 1.2$

Aspromonte Unit

VER $1 \mathrm{~ms}$ (3.3 mg); J=2.938* 10-3; $\mathrm{Ar} *=83,820 ; \mathrm{K}=92.0 \%$; $\mathrm{Ca}=0.4 \% ; \mathrm{Cl}=81 \mathrm{ppm}$

\begin{tabular}{|c|c|c|c|c|c|c|c|c|c|c|c|c|c|}
\hline 1 & 607 & $2,977.42$ & \pm 0.26 & $43.187 \pm$ & \pm 0.091 & 2.12 & \pm 0.05 & $1.11 \pm$ & 0.08 & $2.406 \pm$ & 0.04 & $258.8 \pm$ & 1.3 \\
\hline ) & 716 & $2,409.81$ & \pm 0.33 & $52.901 \pm$ & \pm 0.088 & 1.32 & \pm 0.07 & $1.69 \pm$ & 0.07 & 1.546 & 0.07 & 185.6 & 3 \\
\hline & 741 & $18,508.73$ & \pm 1.61 & 293.02 & \pm 0.27 & 4.52 & \pm 0.07 & $0.77 \pm$ & 0.18 & $1.841 \pm$ & 0.05 & $298.9 \pm$ & 44 \\
\hline
\end{tabular}


Table 5 (continued)

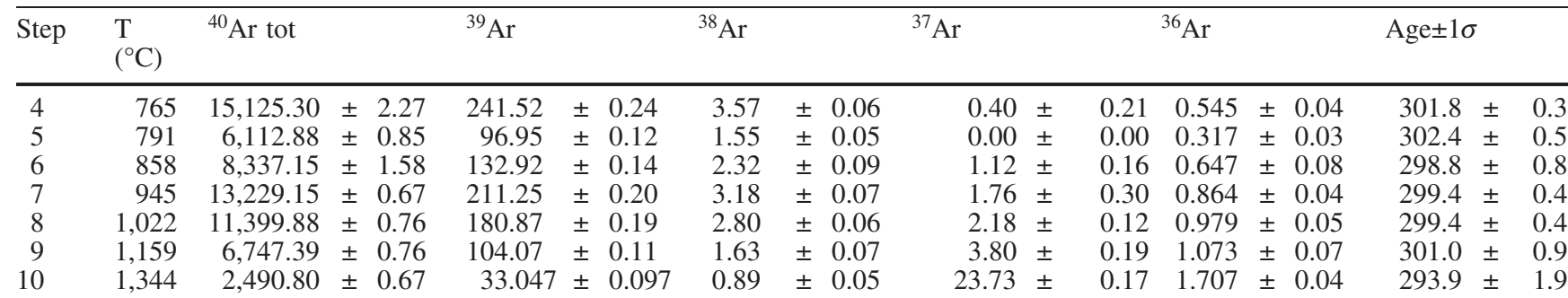

CUM 1 hbl (26.8 mg); J=2.877* 10-3; Ar*=6,370; K=1.4\%o; $\mathrm{Ca}=6.4 \% ; \mathrm{Cl}=206$ ppm

$\begin{array}{rrrl}1 & 713 & 2,425.22 & \pm 0.82 \\ 2 & 921 & 2,216.89 & \pm 0.20 \\ 3 & 960 & 712.22 & \pm 0.06 \\ 4 & 982 & 298.14 & \pm 0.07 \\ 5 & 1,000 & 154.23 & \pm 0.06 \\ 6 & 1,035 & 205.48 & \pm 0.07 \\ 7 & 1,089 & 337.99 & \pm 0.09 \\ 8 & 1,154 & 768.10 & \pm 0.14 \\ 9 & 1,287 & 914.01 & \pm 0.10 \\ 10 & 1,388 & 1,158.20 & \pm 0.49\end{array}$

$15.15 \pm$ $182.20 \pm$ $101.64 \pm$ $36.99 \pm$ $17.36 \pm$ $21.33 \pm$ $41.82 \pm$ $85.33 \pm$ $91.58 \pm$ $108.91 \pm$
$0.115 .401 \pm 0.020 \quad 1609.2 \pm 10.0$ $0.581 .077 \pm 0.011 \quad 1068.8 \pm 2.0$ $0.340 .453 \pm 0.007 \quad 635.5 \pm 2.2$ $0.24 \quad 0.315 \pm 0.006 \quad 666.4 \pm 6.0$ $0.190 .202 \pm 0.006 \quad 697.9 \pm 12.0$ $\begin{array}{llll}0.21 & 0.315 \pm 0.006 & 630.8 \pm 9.4\end{array}$ $0.170 .294 \pm 0.007 \quad 682.7 \pm 4.6$ $\begin{array}{llllll}0.33 & 0.437 & \pm 0.005 & 842.7 \pm 2.3\end{array}$ $0.350 .522 \pm 0.007 \quad 911.4 \pm 2.7$ $\begin{array}{llll}0.35 & 0.715 \pm 0.007 & 889.2 \pm & \pm .9\end{array}$

CUM 3 hbl (31.3 mg); J=2.885* 10-3; Ar*=14,110; K=4.0\%; Ca=6.9\%; Cl=181 ppm

$\begin{array}{rrrrr}1 & 650 & 804.49 & \pm & 0.02 \\ 2 & 713 & 11,656.90 & \pm & 0.58 \\ 3 & 924 & 10,831.76 & \pm & 0.99 \\ 4 & 940 & 6,082.24 & \pm & 0.54 \\ 5 & 962 & 2,153.98 & \pm & 0.14 \\ 6 & 1,000 & 2,475.47 & \pm & 0.38 \\ 7 & 1,037 & 1,499.52 & \pm & 0.16 \\ 8 & 1,087 & 1,962.54 & \pm & 0.20 \\ 9 & 1,152 & 1,812.44 & \pm & 0.28 \\ 10 & 1,286 & 4,408.34 & \pm & 0.42 \\ 11 & 1,388 & 1,082.09 & \pm & 0.38\end{array}$

$\begin{array}{rllll}0.795 & \pm 0.009 & 0.460 & \pm & 0.007 \\ 2.268 & \pm 0.011 & 7.611 & \pm & 0.021 \\ 11.033 & \pm 0.013 & 8.927 & \pm & 0.018 \\ 14.942 & \pm 0.016 & 5.473 & \pm & 0.013 \\ 7.651 & \pm 0.008 & 1.982 & \pm & 0.010 \\ 10.124 & \pm 0.013 & 2.276 & \pm & 0.008 \\ 2.404 & \pm 0.009 & 1.151 & \pm & 0.007 \\ 4.494 & \pm 0.009 & 1.642 & \pm & 0.006 \\ 5.092 & \pm 0.010 & 1.549 & \pm & 0.007 \\ 17.519 & \pm 0.019 & 4.030 & \pm & 0.009 \\ 4.264 & \pm 0.010 & 0.987 & \pm & 0.006\end{array}$

$\begin{aligned} 3.86 & \pm \\ 5.48 & \pm \\ 93.79 & \pm \\ 142.38 & \pm \\ 74.29 & \pm \\ 96.16 & \pm \\ 23.85 & \pm \\ 48.52 & \pm \\ 53.14 & \pm \\ 178.14 & \pm \\ 43.79 & \pm\end{aligned}$

43.79

CUM3 bt $(1.7 \mathrm{mg})$; J=2.894* 10-3; $\mathrm{Ar} *=31,440 ; \mathrm{K}=65.0 \%$; $\mathrm{Ca}=0.8 \% ; \mathrm{Cl}=180 \mathrm{ppm}$

\begin{tabular}{|c|c|c|c|c|c|c|c|c|}
\hline 1 & 541 & $2,919.97$ & \pm 0.88 & 49.38 & \pm 0.11 & 3.16 & \pm 0.11 & 3.51 \\
\hline 2 & 632 & $3,710.54$ & \pm 1.24 & 86.00 & \pm 0.12 & 4.11 & \pm 0.14 & 1.63 \\
\hline 3 & 711 & $7,037.18$ & \pm 1.24 & 203.24 & \pm 0.21 & 6.71 & \pm 0.16 & 1.73 \\
\hline 4 & 787 & $7,799.00$ & \pm 0.88 & 222.25 & \pm 0.25 & 7.01 & \pm 0.10 & 8.30 \\
\hline 5 & 858 & $3,600.74$ & \pm 1.00 & 95.23 & \pm 0.18 & 2.94 & \pm 0.14 & 6.01 \\
\hline 6 & 926 & $3,974.79$ & \pm 0.94 & 96.85 & \pm 0.12 & 3.40 & \pm 0.21 & 8.20 \\
\hline 7 & 1,002 & $5,348.01$ & \pm 1.65 & 135.28 & \pm 0.17 & 3.98 & \pm 0.10 & 20.62 \\
\hline 8 & 1,160 & $3,310.75$ & \pm 0.71 & 73.31 & \pm 0.19 & 2.13 & \pm 0.11 & 9.27 \\
\hline 9 & 1,397 & $1,945.19$ & \pm 0.94 & 17.27 & \pm 0.14 & 0.51 & \pm 0.08 & 7.81 \\
\hline
\end{tabular}

$0.04 \quad 0.624 \pm 0.005$

$0.0437 .688 \pm 0.131$

$0.2730 .899 \pm 0.109$

$0.4112 .356 \pm 0.045$

$0.213 .580 \pm 0.013$

$0.294 .437 \pm 0.017$

$0.114 .019 \pm 0.015$

$0.17 \quad 4.354 \pm 0.017$

$0.17 \quad 2.377 \pm 0.012$

$0.51 \quad 2.655 \pm 0.011$

$0.150 .967 \pm 0.008$
$2130.3 \pm 14.0$
$917.6 \pm 54.0$
$669.4 \pm 11.0$
$700.6 \pm 3.2$
$630.3 \pm 2.0$
$522.6 \pm 2.0$
$579.7 \pm 7.4$
$657.1 \pm 4.2$
$887.6 \pm 2.5$
$851.4 \pm 1.0$
$784.5 \pm 2.3$

MAL 1 hbl (29.4 mg); J=2.852* 10-3; Ar*=2,700; K=2.0\%; $\mathrm{Ca}=7.3 \% ; \mathrm{Cl}=118 \mathrm{ppm}$

\begin{tabular}{|c|c|c|c|c|c|c|c|c|c|c|c|c|c|c|c|c|}
\hline 1 & 714 & 416.54 & \pm 0.04 & $1.634 \pm$ & 0.006 & 0.910 & \pm 0.006 & 28.76 & \pm & 0.14 & 1.192 & \pm & 0.007 & 199.9 & \pm & 5.8 \\
\hline 2 & 922 & 377.02 & \pm 0.04 & $4.937 \pm$ & 0.007 & 2.256 & \pm 0.006 & 102.14 & \pm & 0.32 & 0.282 & \pm & 0.005 & 293.3 & \pm & 1.3 \\
\hline 3 & 962 & 295.40 & \pm 0.03 & $3.822 \pm$ & 0.008 & 1.019 & \pm 0.004 & 76.44 & \pm & 0.26 & 0.173 & \pm & 0.005 & 312.4 & \pm & 1.6 \\
\hline 4 & 981 & 156.53 & \pm 0.04 & $2.032 \pm$ & 0.009 & 0.370 & \pm 0.005 & 37.98 & \pm & 0.19 & 0.037 & \pm & 0.006 & 345.5 & \pm & 3.1 \\
\hline 5 & 999 & 73.69 & \pm 0.01 & $0.999 \pm$ & 0.009 & 0.202 & \pm 0.004 & 18.98 & \pm & 0.17 & 0.086 & \pm & 0.005 & 242.1 & \pm & 4 \\
\hline 6 & 1,036 & 116.89 & \pm 0.02 & $1.562 \pm$ & 0.007 & 0.362 & \pm 0.007 & 31.01 & \pm & 0.17 & 0.056 & \pm & 0.007 & 3.9 & \pm & 3 \\
\hline 7 & 1,069 & 203.50 & \pm 0.03 & $2.816 \pm$ & 0.008 & 0.636 & \pm 0.006 & 55.46 & \pm & 0.21 & 0.082 & \pm & 0.008 & 1.3 & \pm & .0 \\
\hline 8 & 1,108 & 321.51 & \pm 0.05 & $4.292 \pm$ & 0.008 & 0.976 & \pm 0.004 & 85.40 & \pm & 0.28 & 0.117 & \pm & 0.004 & 325.3 & \pm & .2 \\
\hline 9 & 1,149 & 346.03 & \pm 0.06 & 4.492 & 0.009 & 1.091 & \pm 0.010 & 89.71 & \pm & 0.29 & 0.111 & \pm & 0.009 & 337.7 & \pm & .1 \\
\hline 10 & 1,287 & 498.20 & \pm 0.05 & 6.387 & 0.011 & 1.541 & \pm 0.009 & 128.03 & \pm & 0.41 & 0.211 & \pm & 0.005 & 331.4 & \pm & .0 \\
\hline 11 & 1,388 & 612.87 & \pm 0.09 & $7.889 \pm$ & 0.011 & 1.725 & \pm 0.011 & 146.85 & \pm & 0.43 & 0.278 & \pm & 0.005 & 326.3 & \pm & 0.8 \\
\hline \multicolumn{17}{|c|}{ FLO $5 \mathrm{hbl}(33.9 \mathrm{mg}) ; \mathrm{J}=2.825^{*} 10-3 ; \mathrm{Ar} *=2,190 ; \mathrm{K}=1.3 \% \circ ; \mathrm{Ca}=6.8 \% ; \mathrm{Cl}=60 \mathrm{ppm}$} \\
\hline 1 & 710 & 506.39 & \pm & $1.983 \pm$ & 0.005 & 0.816 & 0.005 & 12.92 & \pm & 0.05 & 1.222 & \pm & 0.007 & 342.7 & \pm & 4.7 \\
\hline 2 & 919 & 459.16 & \pm 0.06 & $3.841 \pm$ & 0.007 & 1.338 & \pm 0.006 & 85.18 & \pm & 0.28 & 0.328 & \pm & 0.007 & 438.7 & \pm & .3 \\
\hline 3 & 979 & 650.95 & \pm 0.06 & $6.635 \pm$ & 0.009 & 1.324 & \pm 0.009 & 216.14 & \pm & 0.65 & 0.341 & \pm & 0.007 & 397.7 & \pm & 1.2 \\
\hline 4 & 1,015 & 343.10 & \pm 0.04 & $3.612 \pm$ & 0.011 & 0.552 & \pm 0.009 & 133.17 & \pm & 0.55 & 0.172 & \pm & 0.006 & 391.6 & \pm & 2.0 \\
\hline 5 & 1,042 & 145.52 & \pm 0.04 & $1.546 \pm$ & 0.009 & 0.263 & \pm 0.007 & 49.11 & \pm & 0.30 & 0.111 & \pm & 0.005 & 355.1 & \pm & 0 \\
\hline 6 & 1,063 & 118.65 & \pm 0.05 & $1.161 \pm$ & 0.011 & 0.225 & \pm 0.008 & 37.03 & \pm & 0.37 & 0.061 & \pm & 0.005 & 413.6 & \pm & 5.4 \\
\hline 7 & 1,107 & 136.20 & \pm 0.07 & $1.240 \pm$ & 0.010 & 0.227 & \pm 0.007 & 41.20 & \pm & 0.34 & 0.090 & \pm & 0.006 & 420.5 & \pm & 6.3 \\
\hline 8 & 1,146 & 128.27 & \pm 0.08 & $1.129 \pm$ & 0.005 & 0.267 & \pm 0.009 & 37.01 & \pm & 0.20 & 0.093 & \pm & 0.008 & 424.0 & \pm & 7.9 \\
\hline 9 & 1,281 & 283.20 & \pm 0.07 & $2.614 \pm$ & 0.008 & 0.517 & \pm 0.008 & 79.33 & \pm & 0.33 & 0.192 & \pm & 0.005 & 411.8 & \pm & 2.2 \\
\hline 10 & 1,385 & 180.03 & \pm 0.07 & $1.617 \pm$ & 0.009 & 0.304 & \pm 0.004 & 42.85 & \pm & 0.26 & 0.162 & \pm & 0.004 & 389.6 & \pm & 3.3 \\
\hline
\end{tabular}

$\begin{array}{lllll}0.42 & 5.63 & \pm & 0.11 & 128.1 \pm 3.1\end{array}$ $\begin{array}{llll}0.21 & 3.70 \quad \pm & 0.12\end{array}$ $0.22 \quad 2.33 \pm 0.11$

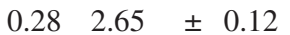
$0.34 \quad 2.24 \quad \pm 0.14$ $0.442 .60 \pm 0.11$ $0.162 .589 \pm 0.065$ $0.361 .94 \pm 0.12$ $0.37 \quad 4.082 \pm 0.094$

$152.3 \pm 2.0$ $156.1 \pm 0.8$ $157.7 \pm 0.8$ $154.4 \pm 2.0$ $165.1 \pm 1.5$ $168.8 \pm 0.7$ $185.2 \pm 2.4$ $210.8 \pm 7.4$ 
Table 5 (continued)

\begin{tabular}{|c|c|c|c|c|c|c|c|c|c|c|c|c|c|c|c|}
\hline Step & $\begin{array}{l}\mathrm{T} \\
\left({ }^{\circ} \mathrm{C}\right)\end{array}$ & ${ }^{40} \mathrm{Ar}$ tot & & ${ }^{39} \mathrm{Ar}$ & & ${ }^{38} \mathrm{Ar}$ & \multicolumn{3}{|c|}{${ }^{37} \mathrm{Ar}$} & \multicolumn{3}{|c|}{${ }^{36} \mathrm{Ar}$} & \multicolumn{3}{|l|}{ Age $\pm 1 \sigma$} \\
\hline \multicolumn{16}{|c|}{ BAD 1 bt $(3.5 \mathrm{mg}) ; \mathrm{J}=2.905^{*} 10-3 ; \mathrm{Ar} *=11,640 ; \mathrm{K}=65.6 \%$; $\mathrm{Ca}=0.1 \% ; \mathrm{Cl}=2,350 \mathrm{ppm}$} \\
\hline 1 & 546 & $2,364.93$ & \pm 0.66 & 91.548 & \pm 0.097 & 17.652 & \pm 0.066 & 0.85 & \pm & 0.09 & 5.114 & \pm 0 & 48.2 & \pm & 0.9 \\
\hline 2 & 630 & $2,272.83$ & \pm 0.91 & 147.37 & \pm 0.17 & 25.141 & \pm 0.086 & 0.87 & \pm & 0.11 & 1.984 & \pm 0.057 & 59.0 & \pm & 0.6 \\
\hline 3 & 712 & $1,371.69$ & \pm 0.54 & 88.93 & \pm 0.10 & 14.675 & \pm 0.054 & 0.36 & \pm & 0.11 & 1.111 & \pm 0.060 & 60.5 & \pm & 1.0 \\
\hline 4 & 763 & 687.77 & \pm 0.40 & 41.004 & \pm 0.086 & 6.689 & \pm 0.043 & 0.29 & \pm & 0.15 & 0.931 & \pm 0.054 & 52.0 & \pm & 2.0 \\
\hline 5 & 834 & 951.21 & \pm 0.31 & 61.407 & \pm 0.071 & 10.171 & \pm 0.071 & 0.07 & \pm & 0.10 & 0.828 & \pm 0.051 & 59.3 & \pm & 1 \\
\hline 6 & 900 & $1,585.47$ & \pm 0.54 & 117.22 & \pm 0.13 & 20.540 & \pm 0.077 & 1.21 & \pm & 0.13 & 1.115 & \pm 0.063 & 55.3 & \pm & 0 . \\
\hline 7 & 970 & 3,98 & \pm 0.46 & 322.40 & \pm 0.29 & 54.68 & \pm 0.13 & 1.35 & \pm & 0.15 & 1. & \pm 0 & 55.9 & \pm & 0 . \\
\hline 8 & 1,023 & $2,050.77$ & \pm 0.66 & 156.52 & \pm 0.1 & 25.537 & \pm 0.091 & 0.95 & \pm & 0.16 & 0.967 & \pm 0. & 58.2 & \pm & 0.4 \\
\hline 9 & 1,094 & 580.93 & \pm 0.49 & 22.162 & \pm 0.054 & 3.601 & \pm 0.040 & 0.64 & \pm & 0.18 & 1.072 & \pm 0.057 & 61.4 & \pm & 3.8 \\
\hline 10 & 1,232 & 541.21 & \pm 0.37 & 3.915 & \pm 0.049 & 0.881 & \pm 0.051 & 1.90 & \pm & 0.13 & 1.382 & \pm 0.049 & 169.7 & \pm & 17.0 \\
\hline 11 & 1,395 & 918.59 & \pm 0.83 & 1.127 & \pm 0.051 & 0.587 & \pm 0.049 & 3.51 & \pm & 0.20 & 3.036 & \pm 0.046 & 98.2 & \pm & 60.0 \\
\hline
\end{tabular}

BAD $1 \mathrm{~ms} ;(3.0 \mathrm{mg}) ; \mathrm{J}=2.909 * 10-3 ; \mathrm{Ar} *=11,300 ; \mathrm{K}=75.6 \%$; $\mathrm{Ca}=2.0 \% ; \mathrm{Cl}=298 \mathrm{ppm}$

$\begin{array}{rrrrr}1 & 597 & 2,334.35 & \pm & 0.11 \\ 2 & 707 & 3,775.47 & \pm & 2.53 \\ 3 & 760 & 5,731.17 & \pm & 0.47 \\ 4 & 807 & 2,349.82 & \pm & 0.37 \\ 5 & 841 & 1,192.13 & \pm & 0.33 \\ 6 & 875 & 997.65 & \pm & 0.57 \\ 7 & 937 & 916.81 & \pm & 0.50 \\ 8 & 1,016 & 1,010.57 & \pm & 0.47 \\ 9 & 1,153 & 1,286.87 & \pm & 0.63 \\ 10 & 1,344 & 2,011.51 & \pm & 1.13\end{array}$

$32.171 \pm 0.0533 .800 \pm 0.060$

$\begin{array}{lllll}176.54 & \pm 0.20 & 5.617 & \pm & 0.087\end{array}$

$524.64 \pm 0.47 \quad 9.618 \pm 0.057$

$185.20 \pm 0.18 \quad 3.334 \pm 0.060$

$65.62 \pm 0.11 \quad 1.411 \pm 0.073$

$35.167 \pm 0.087 \quad 1.303 \pm 0.087$

$37.808 \pm 0.047 \quad 1.004 \pm 0.073$

$51.63 \pm 0.12 \quad 2.048 \pm 0.073$

$53.301 \pm 0.087 \quad 5.429 \pm 0.050$

$33.758 \pm 0.087 \quad 7.505 \pm 0.053$
$2.86 \pm$

$1.81 \pm$

$1.53 \pm$

$1.76 \pm$

$1.14 \pm$

$3.21 \pm$

$4.19 \pm$

$9.49 \pm$

$64.21 \pm$

$81.45 \pm$
$0.18 \quad 7.293 \pm 0.053$

$\begin{array}{lll}0.15 & 6.678 \pm 0.047\end{array}$

$\begin{array}{llll}0.17 & 2.796 & \pm & 0.031\end{array}$

$0.14 \quad 1.885 \pm 0.077$

$\begin{array}{llll}0.22 & 1.739 & \pm & 0.067\end{array}$

$\begin{array}{llll}0.08 & 2.110 & \pm & 0.053\end{array}$

$\begin{array}{llll}0.15 & 2.312 \pm 0.053\end{array}$

$\begin{array}{lll}0.18 & 1.950 \pm 0.047\end{array}$

$0.22 \quad 2.395 \pm 0.043$

$0.325 .776 \pm 0.067$
$29.1 \pm 2.6$

$52.8 \pm 0.4$

$48.4 \pm 0.1$

$50.1 \pm 0.6$

$53.5 \pm 1.6$

$55.0 \pm 2.2$

$32.2 \pm 2.1$

$43.7 \pm 1.4$

$56.6 \pm 1.3$

$47.8 \pm 2.9$

BAD 2 bt $(2.6 \mathrm{mg}) ; \mathrm{J}=2.901 * 10-3 ; \mathrm{Ar} *=10,290 ; \mathrm{K}=60.4 \%$; $\mathrm{Ca}=0.9 \% ; \mathrm{Cl}=114 \mathrm{ppm}$

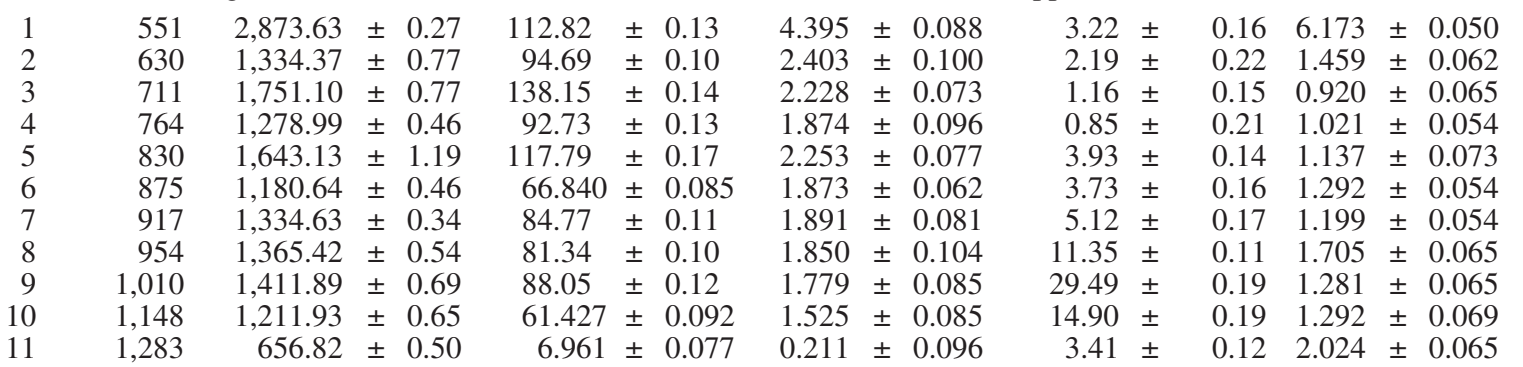

$48.1 \pm 0.7$

$49.2 \pm 1.0$

$55.2 \pm 0.7$

$54.3 \pm 0.9$

$57.2 \pm 0.9$

$61.5 \pm 1.2$

$59.5 \pm 1.0$

$54.7 \pm 1.2$

$60.5 \pm 1.1$

$69.5 \pm 1.7$

$43.7 \pm 14.0$

PER 2 hbl (27.3 mg); J=2.865* 10-3; Ar*=4,310; K=2.9\%o; $\mathrm{Ca}=6.7 \% ; \mathrm{Cl}=133$ ppm

$\begin{array}{rrrrr}1 & 715 & 429.26 & \pm 0.07 \\ 2 & 924 & 435.89 & \pm & 0.10 \\ 3 & 962 & 737.97 & \pm & 0.23 \\ 4 & 1,003 & 409.86 & \pm & 0.04 \\ 5 & 1,028 & 126.35 & \pm & 0.04 \\ 6 & 1,072 & 274.83 & \pm & 0.10 \\ 7 & 1,113 & 463.28 & \pm & 0.04 \\ 8 & 1,150 & 280.59 & \pm & 0.07 \\ 9 & 1,280 & 1,520.88 & \pm & 0.12 \\ 10 & 1,384 & 290.79 & \pm & 0.05\end{array}$

$\begin{array}{rlllllrl}1.757 & \pm & 0.011 & 0.69 & \pm & 0.01 & 9.37 & \pm \\ 5.087 & \pm & 0.008 & 1.66 & \pm & 0.01 & 50.52 & \pm \\ 9.845 & \pm & 0.011 & 1.98 & \pm & 0.01 & 124.30 & \pm \\ 5.584 & \pm & 0.007 & 0.97 & \pm & 0.01 & 68.72 & \pm \\ 1.611 & \pm & 0.005 & 0.33 & \pm & 0.01 & 20.86 & \pm \\ 3.309 & \pm & 0.007 & 0.72 & \pm & 0.01 & 46.39 & \pm \\ 5.405 & \pm & 0.010 & 1.16 & \pm & 0.01 & 71.03 & \pm \\ 3.366 & \pm & 0.009 & 0.65 & \pm & 0.01 & 44.19 & \pm \\ 19.335 & \pm & 0.021 & 3.73 & \pm & 0.01 & 251.05 & \pm \\ 3.579 & \pm & 0.007 & 0.71 & \pm & 0.01 & 44.43 & \pm\end{array}$

$0.07 \quad 0.933 \pm 0.006$

$0.160 .355 \pm 0.005$

$0.360 .181 \pm 0.005$

$0.20 \quad 0.103 \pm 0.011$

$0.090 .049 \pm 0.004$

$0.160 .115 \pm 0.005$

$0.220 .121 \pm 0.005$

$0.170 .096 \pm 0.004$

$0.720 .304 \pm 0.007$

$0.140 .148 \pm 0.005$

$406.5 \pm 4.9$

$313.5 \pm 1.3$

$334.4 \pm 0.7$

$327.3 \pm 2.1$

$334.1 \pm 2.9$

$349.3 \pm 1.9$

$375.7 \pm 1.1$

$357.8 \pm 1.6$

$353.9 \pm 0.5$

$332.2 \pm 1.9$

PER 2 bt $(1.8 \mathrm{mg}) ; \mathrm{J}=2.858 * 10-3 ; \mathrm{Ar}^{*}=36,260 ; \mathrm{K}=70.9 \%$; $\mathrm{Ca}=0.4 \% ; \mathrm{Cl}=236 \mathrm{ppm}$

\begin{tabular}{|c|c|c|c|c|c|c|c|c|c|c|c|c|c|c|c|c|}
\hline 1 & 544 & $1,603.07$ & \pm 0.89 & $30.031 \pm$ & $=0.100$ & 2.45 & \pm 0.09 & 0.53 & \pm & 0.26 & 2.818 & \pm & 0.078 & 127.7 & \pm & 3.7 \\
\hline 2 & 637 & $2,928.10$ & \pm 1.00 & $63.318 \pm$ & $=0.128$ & 2.89 & \pm 0.11 & 3.01 & \pm & 0.32 & 2.553 & \pm & 0.094 & 168.9 & \pm & 2.1 \\
\hline 3 & 715 & $5,464.27$ & \pm 1.39 & $149.942 \pm$ & $=0.178$ & 4.46 & \pm 0.10 & 0.40 & \pm & 0.37 & 1.276 & + & 0.078 & 167.0 & 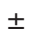 & 8 \\
\hline 4 & 791 & $6,419.56$ & \pm 0.89 & $176.694 \pm$ & 0.189 & 3.59 & \pm 0.16 & 1.24 & \pm & 0.23 & 0.755 & \pm & 0.089 & 72.4 & \pm & 7 \\
\hline 5 & 862 & $4,655.42$ & \pm 1.06 & $118.469 \pm$ & 0.178 & 3.31 & \pm 0.13 & 0.72 & \pm & 0.33 & 1.610 & + & 0.106 & 73.4 & - & 2 \\
\hline 6 & 920 & $6,229.28$ & \pm 1.39 & $167.837 \pm$ & 0.172 & 5.31 & \pm 0.13 & 4.93 & \pm & 0.34 & 2.145 & \pm & 0.144 & 64.3 & \pm & .2 \\
\hline 7 & 003 & $7,588.00$ & \pm 1.11 & 206.997 & 0.239 & 4.60 & \pm 0.08 & 11.62 & \pm & .35 & 1.425 & \pm & 0. & 0.3 & \pm & 6 \\
\hline 8 & 61 & .32 & \pm 0.78 & 128.876 & 0.144 & 3.39 & 0.13 & 6.52 & \pm & 1 & 2.091 & - & 0.067 & 5.1 & - & 0.7 \\
\hline 9 & 1,394 & $1,703.62$ & \pm 1.28 & $11.467 \pm$ & $=0.100$ & 1.15 & \pm 0.08 & 6.21 & \pm & 0.38 & 4.156 & \pm & 0.078 & 2.4 & $I$ & 9.2 \\
\hline \multicolumn{17}{|c|}{ PER $3 \mathrm{hbl}(33.1 \mathrm{mg}) ; \mathrm{J}=2.872 * 10-3 ; \mathrm{Ar} *=2,880 ; \mathrm{K}=1.7 \%$; $\mathrm{Ca}=7.7 \% ; \mathrm{Cl}=33 \mathrm{ppm}$} \\
\hline 1 & 707 & 434.79 & 022 & 1 & 0.006 & 2 & \pm & 35 & \pm & 02 & 22 & \pm & 0.004 & 36.1 & \pm & 3.0 \\
\hline 2 & 918 & 565.00 & \pm 0.045 & $4.824 \pm$ & 0.006 & 0.4 & \pm 0.0 & 59.51 & \pm & 0.18 & 0.448 & \pm & 06 & .3 & \pm & 1.6 \\
\hline 3 & 957 & 418.89 & \pm 0.033 & $3.940 \pm$ & $=0.005$ & 0.389 & \pm 0.008 & 95.08 & \pm & 0.29 & 0.168 & \pm & 0.005 & 443.9 & \pm & 1.5 \\
\hline 4 & 997 & 704.76 & \pm 0.069 & $8.709 \pm$ & 0.009 & 0.785 & \pm 0.010 & 260.09 & \pm & 0.73 & 0.204 & \pm & 0.005 & 363.9 & \pm & 0.6 \\
\hline 5 & 1,022 & 277.89 & \pm 0.036 & $3.701 \pm$ & 0.007 & 0.305 & \pm 0.006 & 95.17 & \pm & 0.31 & 0.096 & \pm & 0.005 & 332.9 & \pm & 1.3 \\
\hline 6 & 1,064 & 100.25 & \pm 0.019 & $1.109 \pm$ & 0.006 & 0.089 & \pm 0.007 & 28.26 & \pm & 0.18 & 0.097 & \pm & 0.004 & 320.5 & \pm & 4.8 \\
\hline 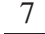 & 1,106 & 135.57 & \pm 0.021 & 1.366 & $=0.010$ & 0.133 & \pm 0.007 & 40.28 & \pm & 0.31 & 0.101 & \pm & 0.006 & 378.5 & \pm & 5.6 \\
\hline
\end{tabular}


Table 5 (continued)

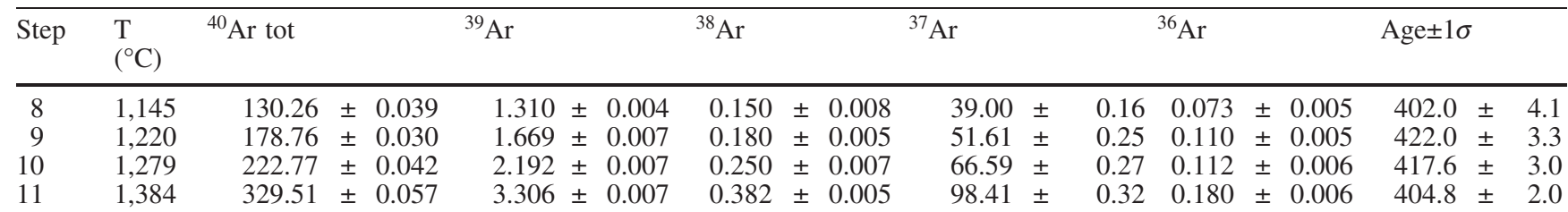

PER 3 bt $(2.5 \mathrm{mg}) ; \mathrm{J}=2.868 * 10-3 ; \mathrm{Ar} *=30,140 ; \mathrm{K}=58.7 \%$; $\mathrm{Ca}=1.4 \% ; \mathrm{Cl}=92 \mathrm{ppm}$

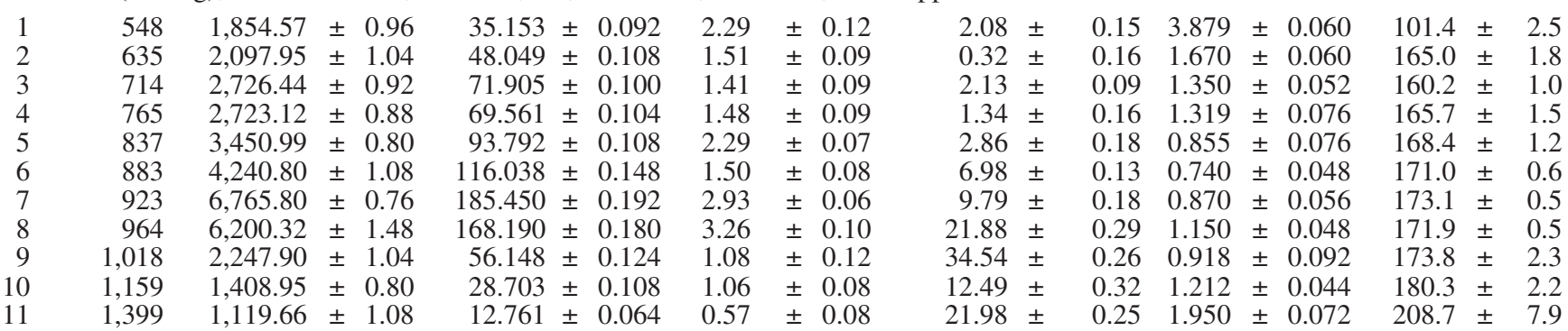

PETR 1 bt $(3.6 \mathrm{mg}) ; \mathrm{J}=2.929 * 10-3 ; \mathrm{Ar} *=30,540 ; \mathrm{K}=73.6 \%$; $\mathrm{Ca}=0.2 \% ; \mathrm{Cl}=275 \mathrm{ppm}$

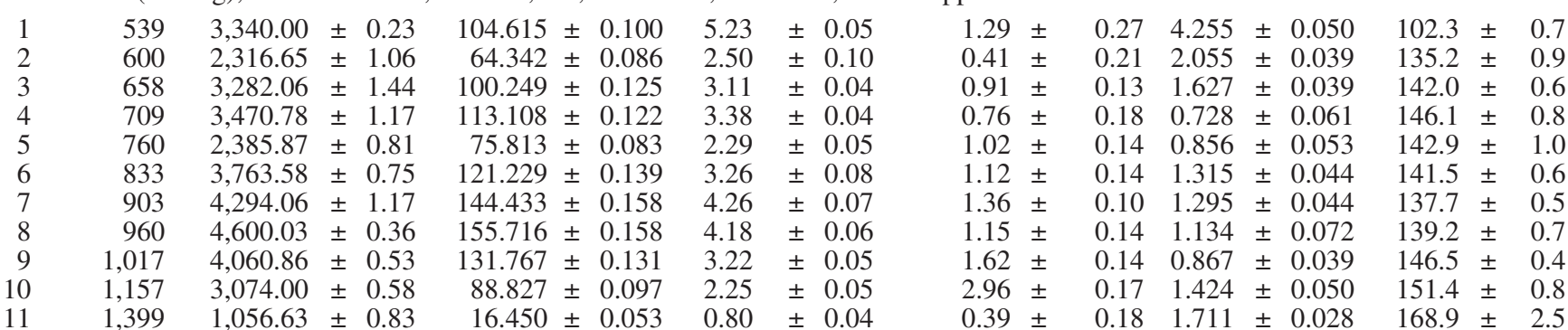

PETR $1 \mathrm{~ms}(2.4 \mathrm{mg}) ; \mathrm{J}=2.934 * 10-3 ; \mathrm{Ar} *=51,050 ; \mathrm{K}=82.7 \%$; $\mathrm{Ca}=0.1 \% ; \mathrm{Cl}=61 \mathrm{ppm}$

\begin{tabular}{|c|c|c|c|c|c|c|c|c|c|c|c|c|c|c|c|c|}
\hline & & & & & & & & & & & & & & & & \\
\hline 1 & 604 & $2,715.34$ & \pm 0.63 & $48.916 \pm$ & \pm 0.121 & 2.99 & \pm 0.07 & 1.81 & \pm & 0.29 & 3.751 & \pm & 0.075 & 166.0 & \pm & 2.2 \\
\hline 2 & 716 & $3,313.49$ & \pm 0.46 & $72.739 \pm$ & \pm 0.088 & 1.82 & \pm 0.10 & 2.32 & 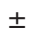 & 0.18 & 2.125 & \pm & 0.104 & 185.6 & \pm & .0 \\
\hline 3 & 745 & $8,350.79$ & \pm 0.79 & $192.752 \pm$ & \pm 0.188 & 3.64 & \pm 0.11 & 1.23 & \pm & 0.22 & 2.084 & \pm & 0.092 & 200.8 & \pm & \\
\hline 4 & 766 & $10,896.13$ & \pm 0.75 & $247.834 \pm$ & \pm 0.246 & 3.88 & \pm 0.09 & 1.38 & \pm & 0.17 & 2.081 & \pm & 0.092 & 07.2 & \pm & \\
\hline 5 & 791 & $6,454.21$ & \pm 1.38 & $145.747 \pm$ & \pm 0.167 & 1.71 & \pm 0.14 & 0.00 & 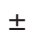 & 0.00 & 0.936 & \pm & 0.058 & 11.5 & & \\
\hline 6 & 822 & $3,693.95$ & \pm 0.83 & $78.686 \pm$ & \pm 0.142 & 1.51 & \pm 0.09 & 0.60 & 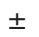 & 0.24 & 1.196 & \pm & $0 .($ & 1.8 & & \\
\hline 7 & 856 & $2,458.65$ & \pm 0.58 & $52.676 \pm$ & \pm 0.079 & .82 & \pm 0.06 & 0.00 & \pm & 0.00 & 0.966 & \pm & 0.079 & 06.2 & & \\
\hline 8 & 940 & $4,175.00$ & \pm 0.39 & $94.251 \pm$ & \pm 0.129 & & \pm 0.07 & 0.91 & \pm & 9 & 63 & \pm & & 5.9 & & \\
\hline 9 & 1,020 & 5.17 & \pm 1.13 & $133.941 \pm$ & \pm 0.154 & & \pm 0.09 & 1.39 & \pm & & & \pm & & 08.2 & & \\
\hline 10 & 1,159 & & \pm 1.33 & $170.577 \pm$ & \pm 0.1 & & \pm 0. & 2.24 & - & & & \pm & & & & \\
\hline 11 & 1,347 & .15 & \pm 0.75 & $16.500 \pm$ & \pm 0.054 & 0.67 & \pm 0.05 & 0.10 & 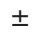 & 26 & 8 & \pm & 0 & 4.2 & & \\
\hline & & ). & $4 *$ & k- & & & $1 \%$ & pm & & & & & & & & \\
\hline 1 & 716 & .49 & \pm 0.13 & $7.996 \pm$ & \pm & 1.0 & \pm 0.0 & 14.80 & \pm & 0.05 & 1.055 & \pm & 7 & 276.4 & \pm & .2 \\
\hline 2 & 922 & 7 & \pm 0.0 & $12.439 \pm$ & \pm & 5 & \pm 0 & 39 & \pm & 8 & 07 & \pm & & .3 & $\perp$ & 0 \\
\hline 3 & 962 & $1,571.93$ & \pm 0.11 & $21.576 \pm$ & \pm 0.020 & 2.947 & \pm 0.0 & 245.88 & \pm & 0.69 & 0.320 & \pm & 0. & .3 & $I$ & .4 \\
\hline 4 & 982 & 668.74 & \pm 0.06 & $9.965 \pm$ & \pm 0.012 & 0.925 & \pm 0.0 & 105.91 & \pm & 0.31 & 0.136 & \pm & 0.005 & 10.0 & & .6 \\
\hline 5 & 999 & 8.61 & \pm 0.03 & $4.391 \pm$ & \pm 0.007 & 0.3 & \pm 0.0 & 43.70 & \pm & 0.14 & 08 & \pm & 08 & 3.9 & \pm & .2 \\
\hline 6 & ,036 & 238.23 & \pm 0.05 & $3.249 \pm$ & \pm 0.009 & 0.3 & \pm 0.0 & 0 & \pm & 0 . & 3 & \pm & 0. & .0 & \pm & \\
\hline 7 & 1,089 & 300.00 & \pm 0.0 & $3.763 \pm$ & \pm 0.008 & 0.4 & \pm 0.0 & 43. & \pm & 5 & 0. & \pm & & & \pm & \\
\hline 8 & 54 & 359.29 & \pm 0.06 & $3.858 \pm$ & \pm 0.011 & 0.4 & \pm 0.0 & 4.77 & \pm & & 5 & \pm & & .0 & \pm & \\
\hline 9 & 83 & & \pm 0.04 & $6 \pm$ & \pm 0.007 & 0.604 & \pm 0.0 & 55 & \pm & 0.17 & & \pm & 05 & & \pm & 1.3 \\
\hline 10 & 1,383 & 607.79 & \pm 0.05 & $6.959 \pm$ & \pm 0.007 & 0.725 & \pm 0.011 & 74.69 & \pm & 0.21 & 0.190 & \pm & 0.006 & 380.7 & \pm & 1.1 \\
\hline 1 & $(3.5$ & ; $J=2.92$ & -3 & $=15,770$ & $=46.0$ & $a=$ & $\mathrm{Cl}=$ & $\mathrm{m}$ & & & & & & & & \\
\hline 1 & 549 & $1,415.28$ & \pm & 52 & \pm & 2.2 & \pm 0.0 & 43.1 & \pm & & & \pm & & 251.6 & \pm & 5.6 \\
\hline 2 & 635 & 9.15 & \pm 0.54 & $1 \pm$ & 7 & 2 & \pm 0 & 2.79 & \pm & 6 & & \pm & & 91.9 & \pm & \\
\hline 3 & 714 & $3,392.83$ & \pm 0.37 & $165.170 \pm$ & \pm 0.160 & 4.07 & \pm 0.04 & 6.29 & \pm & 0.17 & 38 & \pm & 0. & 97.7 & \pm & 5 \\
\hline 4 & 764 & $2,864.71$ & \pm 0.66 & $132.835 \pm$ & \pm 0.177 & 3.69 & \pm 0.04 & 26.68 & \pm & 0.13 & 0.752 & \pm & 0.063 & 102.1 & \pm & 7 \\
\hline 5 & 834 & $1,686.70$ & \pm 0.69 & $70.210 \pm$ & \pm 0.097 & 2.34 & \pm 0.07 & 40.59 & \pm & 0.17 & 0.971 & \pm & 40 & 102.4 & \pm & 8 \\
\hline 6 & 883 & $1,072.04$ & \pm 0.37 & $36.794 \pm$ & \pm 0.063 & 1.44 & \pm 0.04 & 22.87 & \pm & 0.18 & 1.218 & \pm & 9 & 99.6 & \pm & 1 \\
\hline 7 & 925 & $1,326.40$ & \pm 0.51 & $45.423 \pm$ & \pm 0.083 & 1.63 & \pm 0.06 & 45.13 & \pm & 0.27 & 0.905 & \pm & 54 & 119.4 & \pm & 0 \\
\hline 8 & 963 & $1,702.49$ & \pm 0.54 & $53.097 \pm$ & \pm 0.080 & 2.13 & \pm 0.06 & 91.52 & \pm & 0.34 & 0.954 & \pm & 37 & 136.6 & \pm & 0 \\
\hline 9 & 1,022 & ,302.57 & \pm 0.54 & $42.360 \pm$ & \pm 0.074 & 1.36 & \pm 0.06 & 35.28 & \pm & 0.23 & 0.819 & \pm & 0.060 & 127.8 & \pm & \\
\hline 10 & 1,160 & $1,298.27$ & \pm 0.71 & $29.439 \pm$ & \pm 0.040 & 1.18 & \pm 0.07 & 35.35 & \pm & 0.15 & 1.640 & \pm & 0.034 & 140.7 & \pm & 3.9 \\
\hline 11 & 1,396 & $1,212.35$ & \pm 0.74 & $10.425 \pm$ & \pm 0.043 & 0.93 & \pm 0.08 & 23.63 & \pm & 0.16 & 3.099 & \pm & 0.049 & 145.1 & \pm & 4.9 \\
\hline
\end{tabular}


Table 5 (continued)

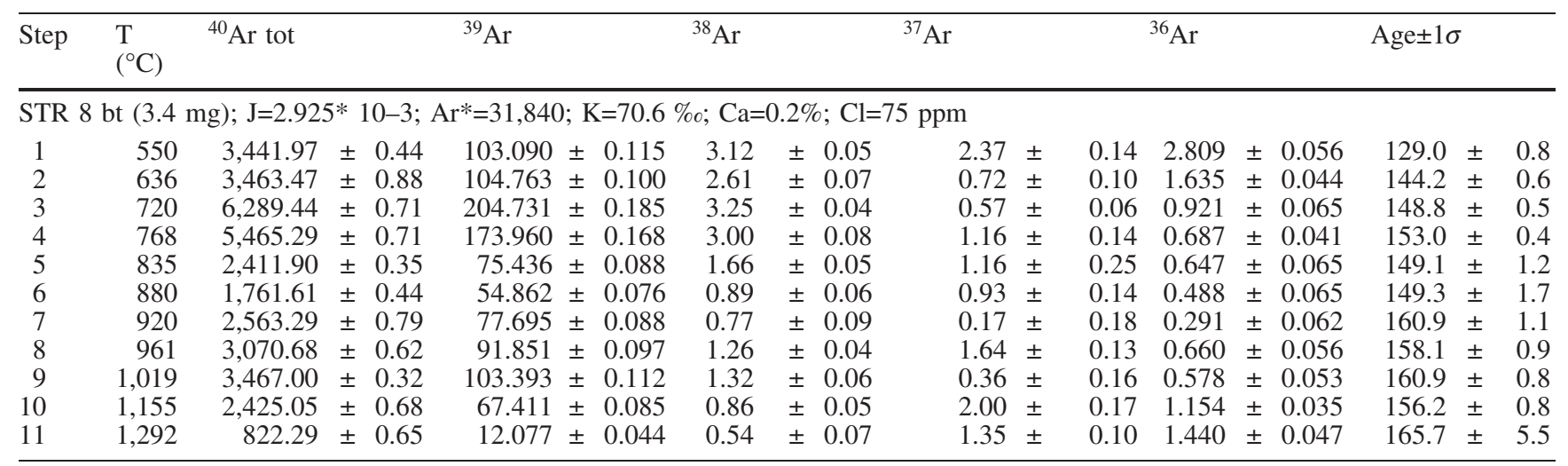

